\title{
ON THE TOPOLOGICAL DYNAMICS AND PHASE-LOCKING RENORMALIZATION OF LORENZ-LIKE MAPS
}

\author{
LL. ALSEDÀ AND A. FALCÓ
}

\begin{abstract}
The aim of this paper is twofold. First we give a characterization of the set of kneading invariants for the class of Lorenz-like maps considered as a maps of the circle of degree one with one discontinuity. In a second step we will consider the subclass of the Lorenz-like maps generated by the class of Lorenz maps in the interval. For this class of maps we give a characterization of the set of renormalizable maps with rotation interval degenerate to a rational number, that is, of phase-locking renormalizable maps. This characterization is given by showing the equivalence between the geometric renormalization procedure and the combinatorial one (which is expressed in terms of an $*$-like product defined in the set of kneading invariants). Finally, we will prove the existence, at a combinatorial level, of periodic points of all periods for the renormalization map.
\end{abstract}

\section{INTRODUCTION}

An important question in the theory of dynamical systems is whether small scale geometric properties of a dynamical system are determined by the combinatorial properties of the system. In the case of unimodal maps such universality was discovered by Coullet and Tresser and Feigenbaum independently. In both cases renormalization ideas that arose from statistical physics were used. The idea is to study dynamical or parameter scaling laws by iterating certain renormalization operator acting in the space of dynamical systems. This operator acts as a microscope: the image under renormalization is another map in the class of maps under consideration which describes the geometry and dynamics on a smaller scale. In the case of unimodal maps the universality was understood by conjecturing that the renormalization operator has a unique hyperbolic fixed point whose invariant manifolds have certain "good" properties.

On the other hand, in the recent years some attention has been paid to Lorenz maps and $\beta$-transformations due to the fact that they help in understanding the dynamics of important three dimensional flows (see [22] and [18]). Lorenz maps were obtained by Lorenz when studying geometric models of the Lorenz equations (see [13], [10], [11], [21] and [22]). A Lorenz map is a map $f$ from the unit interval of the real line into itself which has the following three properties (see Figure 1(a)).

(1) $f$ is differentiable and monotonic for $x \neq c, c \in(0,1)$;

Date: June 14, 2000.

2000 Mathematics Subject Classification. Primary 37E10, 37E20.

Key words and phrases. Lorenz maps, circle maps, Kneading theory.

This work has been partially supported by the DGES grant number PB96-1153 and by the grant number INV98-CB-12-89 from the Direcció General d'Ensenyaments Universitaris i Investigació de la Generalitat Valenciana. 


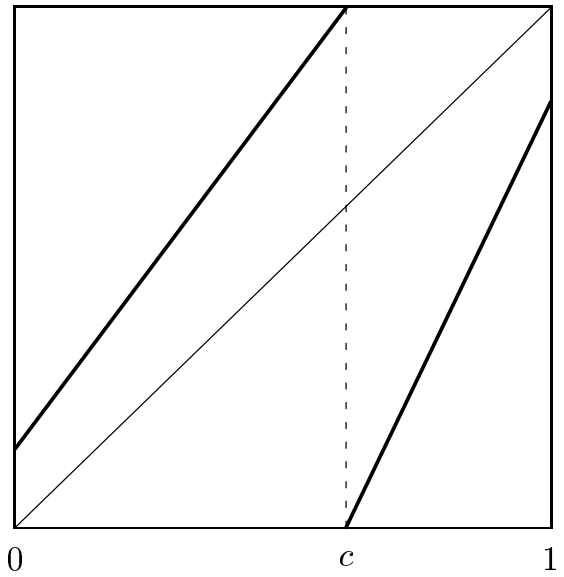

(a)

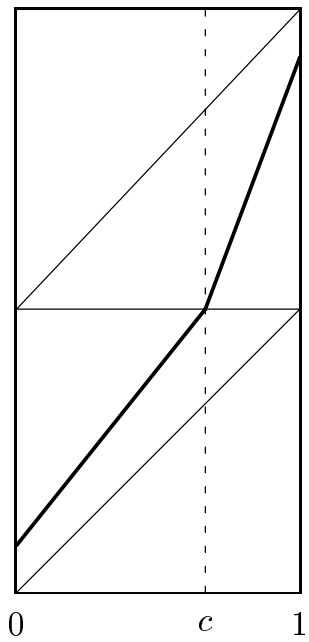

(b)

Figure 1. A Lorenz map (a) and its representation as a degree one lifing (b).

(2) $\lim _{x \nearrow_{c}} f(x)=1, \lim _{x \searrow} f(x)=0$ and $f(c)=0$;

(3) there exists $\varepsilon>0$ such that $f^{\prime}(x) \geq 1+\varepsilon$ for $x \neq c$.

Also, a map verifying (1), (2) and being topologically expansive (see [12] for a definition) is called a topologically expansive Lorenz map. The topological dynamics of such maps as well as more general classes of maps (still verifying (1) and (2)) have been studied extensively in the literature (see [8], [9], [12], [15] and [18]).

We observe that if $f$ is a Lorenz map then the map $F: \mathbb{R} \longrightarrow \mathbb{R}$ defined by

$$
\left.F\right|_{[0,1)}(x)= \begin{cases}f(x) & \text { if } x \in[0, c), \\ f(x)+1 & \text { if } x \in[c, 1) ;\end{cases}
$$

and $F(x)=F(x-E(x))+E(x)$, where $E(\cdot)$ denotes the integer part function (see Figure 1 ) is the lifting of a (discontinuous) circle map of degree one. This map allows us to use the whole theory of rotation numbers for degree one circle maps in the study of the Lorenz maps. This is, precisely, the framework developed in [3]. Following this strategy, in this paper, we will formalize the Lorenz maps as a subclass of the class of Lorenz-like maps (see Section 2 for a precise definition). The class of Lorenz maps extends the Lorenz maps on the interval and the topologically expansive Lorenz maps, while reformulating them as (discontinuous) degree one circle maps.

The main goal of this paper is twofold. First, by using a Kneading Theory, we give a classification, of the Lorenz-like maps as dynamical systems. We recall that this technique was introduced essentially by Milnor and Thurston [16] and it is based in the notion of the kneading invariant. The set of all these kneading invariants provides a representation of all maps in the class under consideration as dynamical systems at the symbolic level. The study of the topological dynamics is often much 
easier when done by using the symbolic representation of the maps as kneading invariants instead of the maps themselves. In [12] this strategy was used to provide a topological classification of the topologically expansive Lorenz maps. For this class of maps, a characterization of the set of all kneading pairs was obtained ([12, Theorem 1]) and the dynamics of each map in the class was completely described by characterizing the set of all itineraries of the map in terms of its kneading pair ([12, Theorem $\left.\left.2^{*}\right]\right)$. The first main result of this paper is an extension of $[12$, Theorem 1] to the Lorenz-like maps (see Theorem A in Subsection 2.1). This is extension is obtained as a consequence of the proof of the main result of [1]. To prove Theorem A we will use the coding introduced by Alsedà and Mañosas in [5] which is an extension of the Kneading Theory for continuous bimodal degree one circle maps introduced by the same authors in [4]. This coding, in the case of the Lorenz maps, is the natural one used by Hubbard and Sparrow in [12]. However we note that some progress in the above direction has been done already. Indeed, in [5], Theorem $2 *$ of [12] was extended to the class of Lorenz-like maps (see Proposition 4) and the rotation interval of such maps was characterized in terms of the kneading pair (see Corollary 5).

The second goal of this paper is to extend the renormalization theory to the class of Lorenz maps. The first step towards this end is the following. For each rational number $0<a<1$ we introduce an $*$-like product (see [6]), denoted by $a \odot$, acting on the set of kneading pairs of the Lorenz maps. The main properties of the $a \odot$ product are given by Theorem B which is the second main result of this paper. It is stated in Subsection 2.3. Afterwards, the notion of renormalizable Lorenz map is introduced for the class of Lorenz maps whose rotation interval is degenerate to a rational in $(0,1)$. Theorem $\mathrm{C}$, which is the last main result of the paper, gives a characterization of the renormalizable Lorenz maps. Essentially, a Lorenz map is renormalizable if and only if its kneading invariant is $a \odot$ times the kneading invariant of another Lorenz map. Thus the $a \odot$ product is the symbolic version of the geometric renormalization operator and, hence, the renormalization domain of the class of Lorenz maps is characterized as the image of the $a \odot$ product for some $a$. Theorem $\mathrm{C}(\mathrm{c})$ is devoted to show the existence of periodic points of any combinatorial type for the renormalization operator (at the kneading invariants level). We remark that this result is the analogue (at the symbolic level) of Martens Theorem for unimodal maps (see [14]).

This paper is organized as follows. In Section 2 we will introduce the necessary notation, state all the main results of the paper and prove Theorem A. Section 3 is devoted to prove Theorem B. Finally, Theorem C is proved in Section 4.

\section{Definitions AND STATEMENTS OF THE MAIN RESUlts}

Given a map $F: \mathbb{R} \longrightarrow \mathbb{R}$ we denote $\lim _{y \downarrow x} F(y)$ by $F\left(x^{+}\right)$and $\lim _{y \uparrow x} F(y)$ by $F\left(x^{-}\right)$, if they exist.

Now, we introduce the class $\mathcal{L}$ of Lorenz-like maps as follows (see Figure 2). We say that $F \in \mathcal{L}$ if:

(i) $F(x+1)=F(x)+1$ for each $x \in \mathbb{R}$.

(ii) $\left.F\right|_{(0,1)}$ is bounded, continuous, non-decreasing and $F\left(1^{-}\right) \geq F\left(0^{+}\right)+1$.

We observe that, when defining the class $\mathcal{L}$ we have removed the assumption that $F\left(0^{+}\right) \in[0,1)$. This is not relevant since the map $F-E\left(F\left(0^{+}\right)\right)$also belongs 


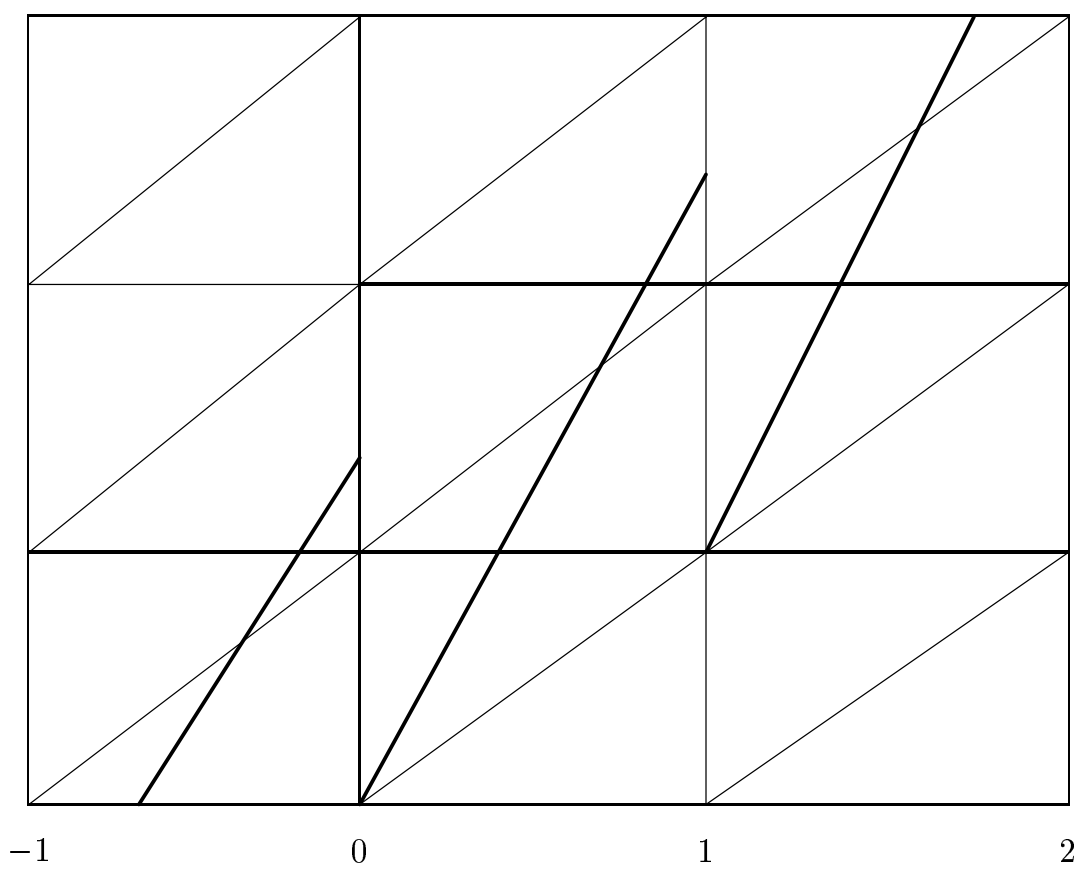

Figure 2. A Lorenz-like map.

to $\mathcal{L}$ and verifies this restriction. Also note that $F(0)$ is not determined by the above conditions. So, in what follows we will assume that $F(0)$ is $F\left(0^{+}\right), F\left(0^{-}\right)$, or both, if necessary. On the other hand, we are not imposing any condition on the derivative of $F$ since for our purposes this will be irrelevant).

ALorenz map will be a map $F$ from $\mathcal{L}$ such that $F\left(0^{+}\right) \in[0,1)$ and $F\left(1^{-}\right) \leq$ $F\left(1^{+}\right)+1$. The class of all Lorenz maps will be denoted by $\mathcal{L}$ m. As it has been said before, the class of Lorenz maps extends the (interval) Lorenz maps and the topologically expansive Lorenz maps.

2.1. A characterization of the kneading pair for Lorenz-like maps. Now, we follow [5] to introduce the coding for the class $\mathcal{L}$. For $F \in \mathcal{L}, x \in \mathbb{R}$ and $i \geq 1$, let $d_{i}=E\left(F^{i}(x)\right)-E\left(F^{i-1}(x)\right)$. Then the reduced itinerary of $x$, denoted by $\widehat{\underline{I}}_{F}(x)$, is defined as

$$
\begin{cases}d_{1} d_{2} \ldots & \text { if } D\left(F^{i}(x)\right) \neq 0 \text { for all } i \geq 1 \\ d_{1} d_{2} \ldots d_{n} & \text { if } D\left(F^{i}(x)\right) \neq 0 \text { for } i \in\{1, \ldots, n-1\} \text { and } D\left(F^{n}(x)\right)=0,\end{cases}
$$

where $D(z)$ denotes the function $z-E(z)$. Note that, since $F \in \mathcal{L}$ we have $\widehat{I}_{F}(x)=$ $\widehat{\underline{I}}_{F}(x+k)$ for all $k \in \mathbb{Z}$. Observe also that $\widehat{\underline{I}}_{F}(0)$ is the empty sequence.

Let $\underline{\alpha}=\alpha_{1} \alpha_{2} \ldots$ be a finite or infinite sequence of integers. We say that $\underline{\alpha}$ is admissible if it is either finite (or empty) or infinite and there exists $k \in \mathbb{N}$ such that $\left|\alpha_{i}\right| \leq k$ for all $i \geq 1$. The set of admissible sequences will be denoted by $\mathcal{A D}$. Notice that any reduced itinerary is an admissible sequence. 
Now we shall introduce some notation for admissible sequences (and hence for reduced itineraries). The cardinality of an admissible sequence $\underline{\alpha}$ will be denoted by $|\underline{\alpha}|$ (if $\underline{\alpha}$ is infinite we write $|\underline{\alpha}|=\infty$ ). We denote by $S$ the shift operator which acts on the set of admissible sequences of length greater than one as follows: $S(\underline{\alpha})=\alpha_{2} \alpha_{3} \ldots$ if $\underline{\alpha}=\alpha_{1} \alpha_{2} \ldots$ We will write $S^{k}$ for the $k$-th iterate of $S$. Obviously $S^{k}$ is only defined for admissible sequences of length greater than $k$. Clearly, for each $x \in \mathbb{R}$ we have $S^{n}\left(\widehat{\underline{I}}_{F}(x)\right)=\widehat{\underline{I}}_{F}\left(F^{n}(x)\right)$ if $\left|\widehat{\underline{I}}_{F}(x)\right|>n$.

Let $\underline{\alpha}=\alpha_{1} \alpha_{2} \ldots \alpha_{n}$ and $\underline{\beta}=\beta_{1} \beta_{2} \ldots$ be two sequences of integers. We shall write $\underline{\alpha} \underline{\beta}$ to denote the concatenation of $\underline{\alpha}$ and $\underline{\beta}$ (that is, to denote the sequence $\left.\alpha_{1} \alpha_{2} \ldots \alpha_{n} \beta_{1} \beta_{2} \ldots\right)$. We also shall use the notation $\underline{\alpha}^{n}$ to denote the concatenation of $\underline{\alpha} n$ times and $\underline{\alpha}^{\infty}$ to denote $\underline{\alpha \alpha} \ldots$.

Now we endow the set of admissible sequences with a total ordering. Let $\underline{\alpha}=$ $\alpha_{1} \alpha_{2} \ldots$ and $\beta=\beta_{1} \beta_{2} \ldots$ be two admissible sequences such that $\underline{\alpha} \neq \beta$. We say that $\underline{\alpha}<\underline{\beta}$ if either there exists $n$ such that $\alpha_{i}=\beta_{i}$ for $i=1,2, \ldots, n-1$ and $\alpha_{n}<\beta_{n}$ or $|\underline{\alpha}|=n-1$ and $\beta=\alpha_{1} \alpha_{2} \ldots \alpha_{n-1} \beta_{n} \beta_{n+1} \ldots$.

To define the kneading pair of a map $F \in \mathcal{L}$ we introduce the following notation. For a point $x \in \mathbb{R}$ we define the sequences $\widehat{\underline{I}}_{F}\left(x^{+}\right)$and $\widehat{\underline{I}}_{F}\left(x^{-}\right)$as follows. For each $n \geq 0$ there exists $\delta(n)>0$ such that $d\left(F^{n-1}(y)\right)$ takes constant value for each $y \in(x, x+\delta(n))$ (respectively, $y \in(x-\delta(n), x)$ ). Denote this value by $d\left(F^{n-1}\left(x^{+}\right)\right)$(respectively, $d\left(F^{n-1}\left(x^{-}\right)\right)$). Then we set

$$
\widehat{\underline{I}}_{F}\left(x^{+}\right)=d\left(x^{+}\right) d\left(F\left(x^{+}\right)\right) \ldots \quad \text { and } \quad \widehat{I}_{F}\left(x^{-}\right)=d\left(x^{-}\right) d\left(F\left(x^{-}\right)\right) \ldots .
$$

Clearly, $\widehat{\underline{I}}_{F}\left(x^{+}\right)$and $\widehat{\underline{I}}_{F}\left(x^{-}\right)$are infinite admissible sequences and, $\widehat{\underline{I}}_{F}\left(x^{+}\right)=\widehat{\underline{I}}_{F}((x+$ $\left.k)^{+}\right)$and $\widehat{I}_{F}\left(x^{-}\right)=\underline{\underline{I}}_{F}\left((x+k)^{-}\right)$for all $k \in \mathbb{Z}$. Moreover, for each $x \in \mathbb{R}$ we have $\widehat{I}_{F}\left(x^{-}\right) \leq \widehat{\widehat{I}}_{F}(x) \leq \widehat{\widehat{I}}_{F}\left(x^{+}\right)$and if $\left|\widehat{I}_{F}(x)\right|=\infty$ then $\widehat{\underline{I}}_{F}\left(x^{+}\right)=\widehat{I}_{F}\left(x^{-}\right)=\widehat{I}_{F}(x)$. We note that for each $x \in \mathbb{R}$ we have $\underline{\underline{I}}_{F}\left(x^{ \pm}\right)=d\left(x^{ \pm}\right) \widehat{I}_{F}\left(\left(F\left(x^{ \pm}\right)\right)^{ \pm}\right)$. Consequently, for each $n \in \mathbb{N}$ we have

$$
\widehat{\underline{I}}_{F}\left(x^{ \pm}\right)=d\left(x^{ \pm}\right) d\left(F\left(x^{ \pm}\right)\right) \cdots d\left(F^{n-1}\left(x^{ \pm}\right)\right) \widehat{\underline{I}}_{F}\left(\left(F^{n}\left(x^{ \pm}\right)\right)^{ \pm}\right) .
$$

Let $F \in \mathcal{L}$. The pair $\left(\widehat{I}_{F}\left(0^{+}\right), \widehat{I}_{F}\left(0^{-}\right)\right)$will be called the kneading pair of $F$ and will be denoted by $\mathcal{K}(F)$. Clearly, for each $F \in \mathcal{L}$ we have $\mathcal{K}(F) \in \mathcal{A D} \times \mathcal{A D}$.

Let $\underline{\alpha}, \beta, \underline{\nu} \in \mathcal{A D}$ be such that $\underline{\alpha}$ and $\underline{\beta}$ are infinite. We will say that $\underline{\nu}$ is quasidominated by $(\underline{\alpha}, \underline{\beta})$ if and only if

$$
\underline{\alpha} \leq S^{n}(\underline{\nu}) \leq \underline{\beta}
$$

for all $n<|\underline{\alpha}|$. We also will say that $\underline{\nu}$ is dominated by $(\underline{\alpha}, \underline{\beta})$ if and only if $\underline{\nu}$ is quasidominated by $(\underline{\alpha}, \beta)$ and the above inequalities are strict. Finally we will say that $\underline{\nu}$ is quasidominated (respectively dominated) by $F$ if it is quasidominated (respectively dominated) by $\left(\widehat{I}_{F}\left(0^{+}\right), \widehat{I}_{F}\left(0^{-}\right)\right)$.

The next result, due to Alsedà and Mañosas [5, Proposition 4], characterizes the set of reduced itineraries (and hence the dynamics) of a map $F \in \mathcal{L}$ in terms of the kneading pair. It extends $\left[12\right.$, Theorem $\left.2^{*}\right]$ to the class $\mathcal{L}$.

Proposition 2.1. For $F \in \mathcal{L}$ the following statements hold.

(a) If $x \in \mathbb{R} \backslash \mathbb{Z}$ then $\widehat{I}_{F}(x)$ is quasidominated by $F$.

(b) If $\underline{\alpha} \in \mathcal{A D}$ is dominated by $F$ then there exists $x \in(0,1)$ such that $\widehat{I}_{F}(x)=$ $\underline{\alpha}$. 
From this proposition it arises naturally the question of characterizing the set of all kneading pairs of all maps from class $\mathcal{L}$. The following result, which follows from [1, Proposition 3.3], gives a first approach to this problem.

Proposition 2.2. For each $F \in \mathcal{L}$ we have that $\widehat{I}_{F}\left(0^{+}\right)$and $\widehat{I}_{F}\left(0^{-}\right)$are quasidominated by F.

To characterize the pairs of admissible sequences that can occur as a kneading pair of a map from $\mathcal{L}$ we will define a set $\mathcal{E} \subset \mathcal{A D} \times \mathcal{A D}$ which turns to be the set of all kneading pairs of maps from $\mathcal{L}$. To define the set $\mathcal{E}$ we introduce the following notation.

Let $\underline{\alpha}=\alpha_{1} \alpha_{2} \ldots$ be an admissible sequence. We will denote by $\underline{\alpha}^{\prime}$ the sequence $\left(\alpha_{1}+1\right) \alpha_{2} \ldots$. Now, let $\mathcal{E}^{*}$ be the set of all pairs $\left(\underline{\nu}_{1}, \underline{\nu}_{2}\right) \in \mathcal{A D} \times \mathcal{A D}$ such that $\left|\underline{\nu}_{i}\right|=\infty$ for $i=1,2$ and the following conditions hold:

(KP1) $\underline{\nu}_{1}^{\prime}<\underline{\nu}_{2}$.

(KP2) $\underline{\nu}_{i}$ is quasidominated by $\left(\underline{\nu}_{1}, \underline{\nu}_{2}\right)$ for all $i \in\{1,2\}$.

Condition (KP2) says, in particular, that $\underline{\nu}_{1}$ and $\underline{\nu}_{2}$ are minimal and maximal, respectively, according to the following definition. For $\underline{\alpha} \in \mathcal{A D}$ we say that $\underline{\alpha}$ is minimal (respectively, maximal) if and only if $\underline{\alpha} \leq S^{n}(\underline{\alpha})$ (respectively, $\underline{\alpha} \geq S^{n}(\underline{\alpha})$ ) for all $n<|\underline{\alpha}|$.

As we will see, the above set contains (among others) the kneading pairs of maps from $\mathcal{L}$ with non-degenerate rotation interval. To deal with some special kneading pairs associated to maps with degenerate rotation interval we introduce the following sets.

For $a \in \mathbb{R}$ we set $\varepsilon_{i}(a)=E(i a)-E((i-1) a)$ and $\delta_{i}(a)=\widetilde{E}(i a)-\widetilde{E}((i-1) a)$, where $\widetilde{E}: \mathbb{R} \longrightarrow \mathbb{Z}$ is defined as $E(x)$ when $x \notin \mathbb{Z}$ and $x-1$ otherwise. Also, we set

$$
\widehat{\underline{I}}_{\varepsilon}(a)=\varepsilon_{1}(a) \varepsilon_{2}(a) \ldots \varepsilon_{n}(a) \ldots \quad \text { and } \quad \widehat{\underline{I}}_{\delta}(a)=\delta_{1}(a) \delta_{2}(a) \ldots \delta_{n}(a) \ldots
$$

Set $\underline{\underline{I}}_{\varepsilon}^{*}(a)=\left(\widehat{I}_{\varepsilon}(a)\right)^{\prime}$ and let $\widehat{\underline{I}}_{\delta}^{*}(a)$ denote the sequence which satisfies $\left(\widehat{I}_{\delta}^{*}(a)\right)^{\prime}=$ $\widehat{I}_{\delta}(a)$. Now, for each $a \in \mathbb{R}$, we set

$$
\mathcal{E}_{a}= \begin{cases}\left\{\left(\widehat{I}_{\varepsilon}(a), \widehat{\underline{I}}_{\varepsilon}^{*}(a)\right),\left(\widehat{I}_{\delta}^{*}(a), \widehat{I}_{\delta}(a)\right),\left(\widehat{I}_{\varepsilon}(a), \widehat{I}_{\delta}(a)\right)\right\} & \text { if } a \in \mathbb{Q}, \\ \left.\left\{\widehat{I}_{\delta}^{*}(a), \widehat{\underline{I}}_{\delta}(a)\right)\right\} & \text { otherwise. }\end{cases}
$$

We note that $\mathcal{E}_{a} \cap \mathcal{E}^{*}=\emptyset$ for each $a \in \mathbb{R}$, because condition (KP1) does not hold for any of the elements of $\mathcal{E}_{a}$. Finally we denote by $\mathcal{E}$ the set $\mathcal{E}^{*} \cup\left(\cup_{a \in \mathbb{R}} \mathcal{E}_{a}\right)$.

The following result characterizes the kneading pairs of maps from $\mathcal{L}$.

Theorem A. For $F \in \mathcal{L}$ we have $\mathcal{K}(F) \in \mathcal{E}$. Conversely, for each $\left(\underline{\nu}_{1}, \underline{\nu}_{2}\right) \in \mathcal{E}$ there exists $F \in \mathcal{L}$ such that $\mathcal{K}(F)=\left(\underline{\nu}_{1}, \underline{\nu}_{2}\right)$.

Proof. Assume that $F \in \mathcal{L}$ and set $\mathcal{K}(F)=\left(\underline{\nu}_{1}, \underline{\nu}_{2}\right)$. From Proposition 2.2 it follows that (KP2) holds. If (KP1) holds then we are done. Otherwise, we have $\underline{\nu}_{1}^{\prime} \geq \underline{\nu}_{2}$. From [1, Theorem A] it follows that if $\underline{\nu}_{1}^{\prime}=\underline{\nu}_{2}$ then

$$
\left(\underline{\nu}_{1}, \underline{\nu}_{2}\right) \in \begin{cases}\left\{\left(\widehat{I}_{\varepsilon}(a), \widehat{I}_{\varepsilon}^{*}(a)\right),\left(\widehat{I}_{\delta}^{*}(a), \widehat{I}_{\delta}(a)\right)\right\} & \text { if } a \in \mathbb{Q}, \\ \left\{\left(\widehat{I}_{\delta}^{*}(a), \widehat{I}_{\delta}(a)\right)\right\} & \text { otherwise. }\end{cases}
$$

So assume that $\underline{\nu}_{1}^{\prime}>\underline{\nu}_{2}$. Recall that if $F\left(1^{+}\right)=F\left(0^{+}\right)+1<F\left(1^{-}\right)$then $\underline{\nu}_{1}^{\prime} \leq \underline{\nu}_{2}$. Hence, $F\left(1^{+}\right)=F\left(1^{-}\right)$and $F$ is continuous. Then in a similar way to the proof of Theorem 5.1 of $[1]$ it follows that $\mathcal{K}(F) \in \mathcal{E}_{a}$ for some $a \in \mathbb{R}$. 
Now, assume that $\left(\underline{\nu}_{1}, \underline{\nu}_{2}\right) \in \mathcal{E}$. If $\left(\underline{\nu}_{1}, \underline{\nu}_{2}\right) \in \mathcal{E}_{a}$ for some $a \in \mathbb{R}$ then the theorem follows from [1, Theorem 5.1]. So assume that $\left(\underline{\nu}_{1}, \underline{\nu}_{2}\right) \in \mathcal{E}^{*}$. Set $\underline{\nu}_{i}=$ $d_{i, 1} d_{i, 2} \ldots d_{i, k} \ldots$ for $i=1,2$. Since $\underline{\nu}_{1}$ and $\underline{\nu}_{2}$ are admissible there exist $k_{1}, k_{2} \in \mathbb{Z}$ such that $k_{1} \leq d_{i, j} \leq k_{2}$ for all $j \geq 1$ and $i \in\{1,2\}$. Let $F \in \mathcal{L}$ be such that $F\left(0^{+}\right)=k_{1}-1$ and $F\left(1^{-}\right)=k_{2}+1$. Clearly $\mathcal{K}(F)=\left(\left(k_{1}-1\right)^{\infty},\left(k_{2}+1\right)\left(k_{1}-1\right)^{\infty}\right)$ and $\underline{\nu}_{i}$ is dominated by $F$ for $i=1,2$. From Proposition 2.1(b) there exists $x_{i} \in(0,1)$ such that $\widehat{I}_{F}\left(x_{i}\right)=\underline{\nu}_{i}$ for $i=1,2$. By [5, Lemma 1] we have that $0<x_{1}<x_{2}<1$ and $F\left(x_{2}\right)>F\left(x_{1}\right)+1$ because $\underline{\nu}_{1}<\underline{\nu}_{1}^{\prime}<\underline{\nu}_{2}$. Then consider the map $F^{*} \in \mathcal{L}$ defined by

$$
\left.F^{*}\right|_{[0,1]}(x)= \begin{cases}F\left(x_{1}\right) & \text { if } 0<x \leq x_{1} \\ F(x) & \text { if } x_{1} \leq x \leq x_{2} \\ F\left(x_{2}\right) & \text { if } x_{2} \leq x<1\end{cases}
$$

Clearly, $\mathcal{K}\left(F^{*}\right)=\left(\underline{\nu}_{1}, \underline{\nu}_{2}\right)$ and the theorem holds.

To define the ambient space of the set $\mathcal{E}$ we introduce the sets:

$$
\begin{aligned}
& \mathcal{E}_{\varepsilon}=\{\underline{\alpha} \in \mathcal{A D}: \text { there exists } \underline{\beta} \in \mathcal{A D} \text { such that }(\underline{\alpha}, \underline{\beta}) \in \mathcal{E}\}, \text { and } \\
& \mathcal{E}_{\delta}=\{\underline{\beta} \in \mathcal{A D}: \text { there exists } \underline{\alpha} \in \mathcal{A D} \text { such that }(\underline{\alpha}, \underline{\beta}) \in \mathcal{E}\} .
\end{aligned}
$$

The following result characterizes the sets $\mathcal{E}_{\varepsilon}$ and $\mathcal{E}_{\delta}$ (see [7, Theorem 3.1.1]).

Proposition 2.3. The following statements hold.

(a) $\underline{\alpha} \in \mathcal{E}_{\varepsilon}$ if and only if it is minimal.

(b) $\underline{\beta} \in \mathcal{E}_{\delta}$ if and only if it is maximal.

Remark 2.4. The sequence $1^{\infty}$ is the unique minimal sequence starting with 1 and $0^{\infty}$ is the unique maximal one starting with 0 . Therefore, in view of Proposition 2.3, we see that each sequence from $\mathcal{E}_{\varepsilon} \backslash\left\{1^{\infty}\right\}$ (respectively from $\mathcal{E}_{\delta} \backslash\left\{0^{\infty}\right\}$ ) starts with 0 (respectively 1 ).

We consider $\mathcal{E}_{\varepsilon}$ and $\mathcal{E}_{\delta}$ endowed with the order topology and let $\mathcal{E}_{\varepsilon} \times \mathcal{E}_{\delta}$ be with the product topology. We note that $\mathcal{E}$ is strictly contained in $\mathcal{E}_{\varepsilon} \times \mathcal{E}_{\delta}$. To see this consider for example the set $A=\left\{0^{\infty}, 1^{\infty}\right\}$ of admissible sequences. Since

$$
\left((-1)^{\infty}, 0^{\infty}\right),\left(0^{\infty}, 1^{\infty}\right),\left(1^{\infty}, 2^{\infty}\right) \in \mathcal{E},
$$

we have that $A \subset \mathcal{E}_{\varepsilon}$ and $A \subset \mathcal{E}_{\delta}$. In consequence $\left\{\left(0^{\infty}, 1^{\infty}\right),\left(1^{\infty}, 0^{\infty}\right)\right\} \subset \mathcal{E}_{\varepsilon} \times \mathcal{E}_{\delta}$. However, $\left(0^{\infty}, 1^{\infty}\right) \in \mathcal{E}$ but $\left(1^{\infty}, 0^{\infty}\right) \notin \mathcal{E}$.

2.2. Rotation interval, twist periodic orbits and kneading pair. We warn the reader that most of the results we are quoting from other authors will be written in terms of class $\mathcal{L}$ unlike the original versions which are stated for circle maps of degree one.

The notion of rotation number was introduced by Poincaré [19] for homeomorphisms of the circle of degree one. This notion will be used to characterize the set of periods of circle maps of degree one. Let $F \in \mathcal{L}$. For $x \in \mathbb{R}$ we define the rotation number of $x$ as

$$
\limsup _{n \rightarrow \infty} \frac{F^{n}(x)-x}{n}
$$


and we denote it by $\rho(x)$ or $\rho_{F}(x)$. We denote by $R_{F}$ the set of all rotation numbers of $F$. From [17] (see also [20]) it follows that $R_{F}=[a(F), b(F)]$ with

$$
\begin{aligned}
& a(F)=\inf _{x \in \mathbb{R}} \liminf _{n \rightarrow \infty} \frac{F^{n}(x)-x}{n}, \text { and } \\
& b(F)=\sup _{x \in \mathbb{R}} \limsup _{n \rightarrow \infty} \frac{F^{n}(x)-x}{n} .
\end{aligned}
$$

Therefore, in what follows the set $R_{F}$ will be called the rotation interval of $F$.

If $A \subset \mathbb{R}$ and $x \in \mathbb{R}$, we shall write $x+A$ or $A+x$ to denote the set $\{x+a: a \in A\}$. Also, if $B \subset \mathbb{R}$ we shall write $A+B$ to denote the set $\{a+b: a \in A, b \in B\}$.

We shall say that a point $x \in \mathbb{R}$ is periodic (mod. 1) of period $q$ with rotation number $p / q$ for a map $F \in \mathcal{L}$ if $F^{q}(x)-x=p$ and $F^{i}(x)-x \notin \mathbb{Z}$ for $i=1, \ldots, q-1$. A periodic (mod. 1) point of period 1 will be called fixed (mod. 1). Let $F \in \mathcal{L}$ and let $x \in \mathbb{R}$. The set $\left\{F^{n}(x): n \in \mathbb{Z}^{+}\right\}+\mathbb{Z}$ will be called the (mod. 1) orbit of $x$ by $F$. It is not difficult to prove that each point from an orbit (mod. 1) $P$ has the same rotation number. Thus, we can speak about the rotation number of $P$. If $x$ is a periodic (mod. 1) point of $F$ of period $q$ with rotation number $p / q$ then its (mod. 1 ) orbit is called a periodic (mod. 1) orbit of $F$ of period $q$ with rotation number $p / q$. If $P$ is a (mod. 1$)$ orbit of $F$ then we denote by $P_{i}$ the set $P \cap[i, i+1)$ for all $i \in \mathbb{Z}$. Obviously $P_{i}=i+P_{0}$ and, if $P$ has period $q$, then $\operatorname{Card}\left(P_{i}\right)=q$ for all $i \in \mathbb{Z}$.

Let $P$ be a (mod. 1) orbit of a map $F \in \mathcal{L}$. We say that $P$ is a twist orbit if $F$ restricted to $P$ is increasing. If a periodic (mod. 1) orbit is twist then we say that $P$ is a twist periodic orbit. The following result gives a geometrical interpretation of the twist periodic orbits (see for instance [2]).

Lemma 2.5. Let $P=\left\{\ldots, x_{-2}, x_{-1}, x_{0}, x_{1}, x_{2}, \ldots\right\}$ be a twist periodic orbit with period $q$ and rotation number $p / q$. Then $(p, q)=1$. Moreover, if we assume that $x_{i}<x_{j}$ if and only if $i<j$ then $F\left(x_{i}\right)=x_{i+p}$.

The following result, given in [3, Lemma 1], studies the relation between the rotation number and twist orbits.

Lemma 2.6. Let $F \in \mathcal{L}$ and let $a$ be an endpoint of $R_{F}$. Then there exists a twist orbit of $F$ with rotation number a which is contained in a union of closed intervals on which $F$ is increasing. Moreover, if $a=p / q \in \mathbb{Q}$ then this orbit can be chosen periodic (mod. 1) with period $q$.

Now, we will study the basic properties of the sequences $\widehat{\widehat{I}}_{\varepsilon}(a), \widehat{I}_{\delta}(a), \underline{\underline{I}}_{\varepsilon}^{*}(a)$ and $\widehat{I}^{*}(a)$. These sequences give the characterization of the rotation interval by means of the kneading pair. The following result is due to Alsedà and Mañosas (see [5, Theorem 6]).

Theorem 2.7. Let $F \in \mathcal{L}$. Then $R_{F}=[a, b]$ if and only if

$$
\widehat{\underline{I}}_{\delta}^{*}(a) \leq \widehat{I}_{F}\left(0^{+}\right) \leq \widehat{I}_{\varepsilon}(a) \quad \text { and } \quad \widehat{I}_{\delta}(b) \leq \widehat{\underline{I}}_{F}\left(0^{-}\right) \leq \widehat{\underline{I}}_{\varepsilon}^{*}(b) .
$$

2.3. The $\odot$-product: an $*-$ like product for the class of Lorenz maps. In the unimodal framework one of the main tools to explain the renormalization properties is the well-known $*$-product. By using this product it is possible to define, for each periodic kneading sequence $\underline{A}$, a map $\underline{A} *(\cdot)$ from the set of all kneading sequences into itself. Then, if $f$ is a unimodal map having $\underline{A} * \underline{B}$ as a kneading sequence, it follows that $f$ is renormalizable and the renormalization 
operator $\mathcal{R}$ acts in such a way that the kneading sequence of $\mathcal{R}(f)$ is $\underline{B}$. Thus, in some sense the $*$-product is the inverse of the renormalization operator.

The main goal of this section is to construct an $*$-like product (called the $\odot-$ product) for the class of Lorenz maps by using the symbolic properties of the twist periodic orbits.

We recall that $\mathcal{L}$ m denotes the class of all Lorenz maps which consists on all maps $F \in \mathcal{L}$ such that $F\left(0^{+}\right) \in[0,1)$ and $F\left(1^{-}\right) \leq F\left(1^{+}\right)+1$. The set of all admissible sequences of maps from $\mathcal{L}$ m, denoted by $\mathcal{A} \mathcal{D}_{0,1}$, is the set $\Sigma_{2}=\{0,1\}^{\mathbb{N}}$ union the set of finite sequences in the symbols $\{0,1\}$. We take $\mathcal{E}_{\varepsilon}^{*}=\left(\Sigma_{2} \cap \mathcal{E}_{\varepsilon}\right) \backslash\left\{1^{\infty}\right\}$ and $\mathcal{E}_{\delta}^{*}=\left(\Sigma_{2} \cap \mathcal{E}_{\delta}\right) \backslash\left\{0^{\infty}\right\}$. From Proposition 2.3 we have that $\mathcal{E}_{\varepsilon}^{*} \cup\left\{1^{\infty}\right\}$ (respectively, $\mathcal{E}_{\delta}^{*} \cup\left\{0^{\infty}\right\}$ ) are all the minimal (respectively, maximal) sequences in $\Sigma_{2}$.

We endow $\Sigma_{2}$ with the topology defined by the metric

$$
d\left(\left(d_{1} d_{2} \ldots\right),\left(t_{1} t_{2} \ldots\right)\right)=\sum_{i=1}^{\infty} \frac{\left|d_{i}-t_{i}\right|}{2^{i}} .
$$

This topology is compatible with the order topology given by the lexicographical order in $\Sigma_{2}$. With this topology $\Sigma_{2}$ is a compact metric space. Let $S: \Sigma_{2} \longrightarrow \Sigma_{2}$ denote the usual shift transformation restricted to $\Sigma_{2}$. Clearly, $S$ is continuous. Set

$$
\mathcal{E}_{0,1}=\left(\mathcal{E}_{\varepsilon}^{*} \times \mathcal{E}_{\delta}^{*}\right) \cap \mathcal{E}=\left(\mathcal{E} \cap\left(\Sigma_{2} \times \Sigma_{2}\right)\right) \backslash\left\{(\underline{\alpha}, \underline{\beta}): \underline{\alpha}=1^{\infty} \text { or } \underline{\beta}=0^{\infty}\right\} \subset \Sigma_{2} \times \Sigma_{2}
$$

and consider $\mathcal{E}_{0,1}$ endowed with the product topology of $\Sigma_{2} \times \Sigma_{2}$ given by

$$
d^{*}((\underline{\alpha}, \underline{\beta}),(\underline{\nu}, \underline{\kappa}))=\max \{d(\underline{\alpha}, \underline{\nu}), d(\underline{\beta}, \underline{\kappa})\} .
$$

For $a \in \mathbb{R}$ we will denote $a-\widetilde{E}(a)$ by $\widetilde{D}(a)$. Also, $\mathbb{Q} \cap(0,1)$ will be denoted by $\mathbb{Q}^{*}$. To simplify the use of the sequences $\underline{I}_{\varepsilon}(a), \widehat{I}_{\delta}(a), \underline{\underline{I}}_{\varepsilon}^{*}(a)$ and $\widehat{I}_{\delta}^{*}(a)$ the following lemma will be helpful (see [4, (4.1)-(4.3)]).

Lemma 2.8. For any $a \in \mathbb{R}$ the following statements hold.

(a) If $a \notin \mathbb{Z}$ then $\delta_{1}(a)=\varepsilon_{1}(a)+1$. Furthermore, if $a \notin \mathbb{Q}$ then $\delta_{i}(a)=\varepsilon_{i}(a)$ for all $i>1$. That is, $\widehat{\underline{I}}_{\delta}^{*}(a)=\underline{\underline{I}}_{s}(a)$ and $\widehat{\underline{I}}_{\delta}(a)=\underline{\underline{I}}_{\varepsilon}^{*}(a)$. If $a=p / q$ with $(p, q)=1$ and $q>1$ then $\varepsilon_{i}(a)=\delta_{i}(a)$ for $i=2, \ldots, q-1, \delta_{q}(a)=\varepsilon_{q}(a)-1$ and, $\varepsilon_{i+q}(a)=\varepsilon_{i}(a)$ and $\delta_{i+q}(a)=\delta_{i}(a)$ for all $i \in \mathbb{N}$.

(b) If $a \in \mathbb{Z}$ then $\varepsilon_{i}(a)=\delta_{i}(a)=a$ for all $i>0$.

We note that from this lemma it follows that if $a=p / q \in \mathbb{Q}^{*}$ with $(p, q)=1$ and $q>2$ then the finite sequences $\varepsilon_{2}(a) \cdots \varepsilon_{q-1}(a)$ and $\delta_{2}(a) \cdots \delta_{q-1}(a)$ are equal. We will denote this finite sequence by $\underline{r}(a)$. We also define $\underline{r}(1 / 2)$ to be the empty sequence.

Now we are ready to define the $\odot$-operator. Given $d \in\{0,1\}$ we will denote $1-d$ by $\widehat{d}$. Then, for $a \in \mathbb{Q}^{*}$ and $\alpha_{1} \alpha_{2} \cdots \in \mathcal{A D}_{0,1}$, we define

$$
a \odot\left(\alpha_{1} \alpha_{2} \cdots\right)= \begin{cases}\alpha_{1} \underline{r}(a) \alpha_{1} \widehat{\alpha}_{1} \underline{r}(a) \alpha_{2} \widehat{\alpha}_{2} \cdots & \text { if }|\underline{\alpha}|=\infty, \\ \alpha_{1} \underline{r}(a) \alpha_{1} \widehat{\alpha}_{1} \underline{\underline{r}}(a) \alpha_{2} \widehat{\alpha}_{2} \underline{r}(a) \cdots \widehat{\alpha}_{n-1} \underline{r}(a) \alpha_{n} & \text { if }|\underline{\alpha}|=n,\end{cases}
$$

and $\overline{a \odot \underline{\alpha}}=\widehat{\alpha}_{1} S(a \odot \underline{\alpha})$.

Remark 2.9. Let $\underline{\alpha}=\alpha_{1} \alpha_{2} \ldots, \underline{\beta}=\beta_{1} \beta_{2} \ldots \in \mathcal{A D}_{0,1}$ be such that $\alpha_{1}=0$ and $\beta_{1}=1$. Then $(a \odot \underline{\alpha})^{\prime}<a \odot \underline{\beta}$ for each $a \in \mathbb{Q}^{*}$.

The next result, which we will be proved in Section 3, gives a first motivation to the $\odot-$ product. 
Proposition 2.10. Let $a \in \mathbb{Q}^{*}$. Then $a \odot 0^{\infty}=\widehat{I}_{\delta}^{*}(a), \overline{a \odot 1^{\infty}}=\underline{I}_{\varepsilon}(a), \overline{a \odot 0^{\infty}}=$ $\widehat{I}_{\delta}(a)$ and $a \odot 1^{\infty}=\underline{I}_{\varepsilon}^{*}(a)$.

The next theorem and corollary state the main properties of the $\odot$-product.

Theorem B. For any $a \in \mathbb{Q}^{*}$ the following statements hold:

(a) Let $\underline{\alpha}, \underline{\beta} \in \mathcal{A D}_{0,1}$ be such that $\underline{\alpha}<\underline{\beta}$. Then a $\odot \underline{\alpha}<a \odot \underline{\beta}$. Moreover, for each $\underline{\alpha}=\alpha_{1} \alpha_{2} \ldots \in \mathcal{A D}_{0,1}$, we have $\widehat{\underline{I}}_{\delta}^{*}(a) \leq a \odot \underline{\alpha} \leq \underline{\underline{I}}_{\varepsilon}$ (a) if $\alpha_{1}=0$ and $\widehat{I}_{\delta}(a) \leq a \odot \underline{\alpha} \leq \underline{\underline{I}}_{\varepsilon}^{*}(a)$ if $\alpha_{1}=1$.

(b) $a \odot \mathcal{E}_{\varepsilon}^{*} \subset \mathcal{E}_{\varepsilon}^{*}$ and $a \odot \mathcal{E}_{\delta}^{*} \subset \mathcal{E}_{\delta}^{*}$.

(c) If $\underline{\kappa}$ is quasidominated by $(\underline{\alpha}, \underline{\beta}) \in \mathcal{E}_{0,1}$ then $a \odot \underline{\kappa}$ is quasidominated by $(a \odot \underline{\alpha}, a \odot \underline{\beta})$.

Corollary 2.11. Let $a \in \mathbb{Q}^{*}$ and let $(\underline{\alpha}, \underline{\beta}) \in \mathcal{E}_{0,1}$. Then $(a \odot \underline{\alpha}, a \odot \underline{\beta}) \in \mathcal{E}_{0,1} \cap \mathcal{E}^{*}$.

Proof. From the definition of $\mathcal{E}_{0,1}$, Theorem B(b) and Remarks 2.4 and 2.9 it follows that $(a \odot \underline{\alpha}, a \odot \underline{\beta}) \in \mathcal{E}_{\varepsilon}^{*} \times \mathcal{E}_{\delta}^{*}$ and $(a \odot \underline{\alpha})^{\prime}<a \odot \beta$. On the other hand, since $(\underline{\alpha}, \underline{\beta}) \in \mathcal{E}$, from Theorem $\mathrm{A}$ and Proposition 2.2, we get that $\underline{\alpha}$ and $\beta$ are quasidominated by $(\underline{\alpha}, \beta)$. Thus from Theorem $\mathrm{B}(\mathrm{c})$ we see that $a \odot \underline{\alpha}$ and $a \odot \beta$ are quasidominated by $(a \odot \underline{\alpha}, a \odot \underline{\beta})$. Therefore, $(a \odot \underline{\alpha}, a \odot \underline{\beta}) \in \mathcal{E}^{*}$ by definition.

The above corollary motivates the following notation. In what follows, given $a \in \mathbb{Q}^{*}$ and $(\underline{\alpha}, \underline{\beta}) \in \mathcal{E}_{0,1}$, we will denote the kneading pair $(a \odot \underline{\alpha}, a \odot \underline{\beta})$ by $a \odot(\underline{\alpha}, \underline{\beta})$.

Now we will start the study of the renormalization which undergoes from the combinatorial structure of $\mathcal{E}_{0,1}$ given by the $\odot$ product. Let $F \in \mathcal{L} \mathrm{m}$ and assume that $R_{F}=\{p / q\}$ with $p / q \in \mathbb{Q}^{*}$ and $(p, q)=1$. By Lemma 2.6 we know that $F$ has a twist periodic orbit $P$ of period $q$ and rotation number $p / q$. Let $J$ be the closed interval between two consecutive points of $P$ which contains 0 and denote the map $F^{q}-p$ by $G$. One can easily show that:

(i) $G$ fixes the endpoints of $J$

(ii) $G$ is discontinuous at 0 and $G$ is continuous and increasing on the intervals $[\min J, 0)$ and $(0, \max J]$.

(iii) $G\left(0^{-}\right) \geq G\left(0^{+}\right)$(see Figure 3 ).

We will say that the map $F$ is renormalizable if $\min J \leq G\left(0^{+}\right)<0<G\left(0^{-}\right) \leq$ $\max J$ and $G\left(\left[G\left(0^{+}\right), G\left(0^{-}\right)\right]\right) \subset\left[G\left(0^{+}\right), G\left(0^{-}\right)\right]$. In such a case, for $x \in[0,1]$ we define $\varphi_{F}(x)=\left(G\left(0^{-}\right)-G\left(0^{+}\right)\right) x+G\left(0^{+}\right) \in\left[G\left(0^{+}\right), G\left(0^{-}\right)\right]$and $\mathcal{R}_{a}(F) \in \mathcal{L} \mathrm{m}$ by (see Figure 3)

$$
\left.\mathcal{R}_{a}(F)\right|_{[0,1]}(x)= \begin{cases}G\left(\varphi_{F}(x)\right) & \text { if } x \in\left[0, \varphi_{F}^{-1}(1)\right), \\ G\left(\varphi_{F}(x)\right)+1 & \text { if } x \in\left[\varphi_{F}^{-1}(1), 1\right),\end{cases}
$$

and $\mathcal{R}_{a}(F)(x)=\left.\mathcal{R}_{a}(F)\right|_{[0,1]}(x-E(x))+E(x)$. The map $\mathcal{R}_{a}(F)$ will be called the renormalized map of $F$. It turns out that this renormalization process is very well described at a symbolic level by the $\odot$ product as shown by the following theorem.

Given $\underline{\mathbf{a}}=\left(a_{1}, a_{2}, \ldots, a_{n}\right) \in\left(\mathbb{Q}^{*}\right)^{n}$ and $\underline{\alpha} \in \mathcal{A D}_{0,1}$ we will denote $a_{n} \odot\left(\cdots a_{2} \odot\right.$ $\left.\left(a_{1} \odot \underline{\alpha}\right) \cdots\right)$ by $\underline{\mathbf{a}} \odot \underline{\alpha}$. Of course, given $(\underline{\alpha}, \underline{\beta}) \in \mathcal{E}_{0,1}, \underline{\mathbf{a}} \odot(\underline{\alpha}, \underline{\beta})$ stands for $(\underline{\mathbf{a}} \odot$ $\underline{\alpha}, \underline{\mathbf{a}} \odot \underline{\beta})$. Observe that, in view of Corollary 2.11 , $\underline{\mathbf{a}} \odot(\underline{\alpha}, \underline{\beta})$ is well defined and belongs to $\mathcal{E}_{0,1}$. Moreover, if $(\underline{\alpha}, \underline{\beta}) \in \mathcal{E}^{*}$ then, $\underline{\mathbf{a}} \odot(\underline{\alpha}, \underline{\beta})$ also belongs to $\mathcal{E}^{*}$. 


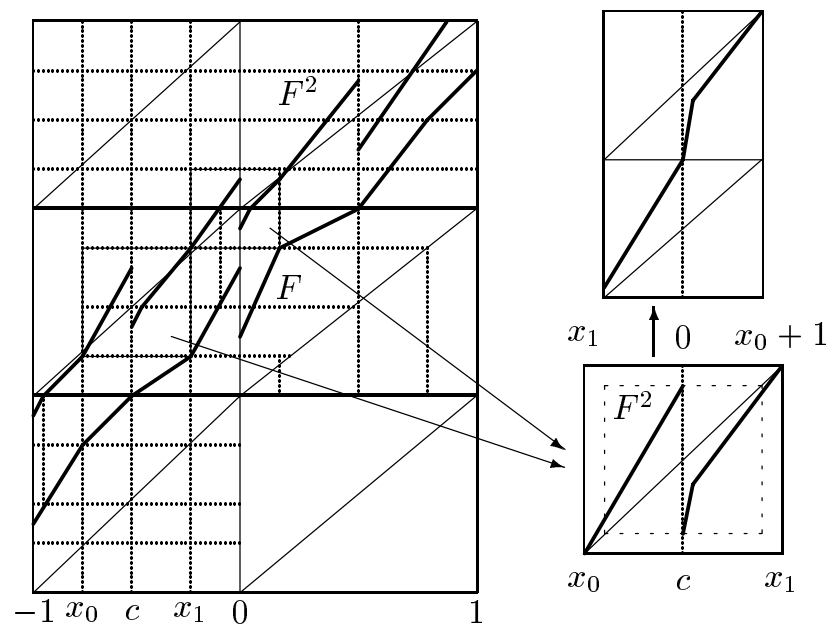

Figure 3. An example of renormalization with $p / q=1 / 2$.

A map $F \in \mathcal{L}$ m will be called symbolically expansive if the map $\widehat{\underline{I}}_{F}(\cdot)$ from $(0,1)$ to $\mathcal{A D}_{0,1}$ is injective. Such a condition is guaranteed by usual topological conditions like $F$ being topologically expansive or having negative Schwarzian derivative.

Theorem C. Let $F \in \mathcal{L} m$ be such that $R_{F}=\{a\}$. Then the following statements hold.

(a) If $F$ is renormalizable then $\mathcal{K}(F)=a \odot \mathcal{K}\left(\mathcal{R}_{a}(F)\right.$ ).

(b) If $F$ is symbolically expansive and $\mathcal{K}(F)=a \odot(\underline{\alpha}, \underline{\beta})$ with $(\underline{\alpha}, \underline{\beta}) \in \mathcal{E}_{0,1}$ then $F$ is renormalizable.

(c) For each $\underline{\mathbf{a}} \in\left(\mathbb{Q}^{*}\right)^{n}$, $\underline{\mathbf{a}} \odot(\cdot)$ is a contractive map from the compact space $\mathcal{E}_{0,1} \cap \mathcal{E}^{*}$ into itself. Therefore, there exists $(\underline{\alpha}, \underline{\beta})_{\mathbf{a}} \in \mathcal{E}_{0,1} \cap \mathcal{E}^{*}$ such that $\underline{\mathbf{a}} \odot(\underline{\alpha}, \underline{\beta})_{\underline{\mathbf{a}}}=(\underline{\alpha}, \underline{\beta})_{\underline{\mathbf{a}}}$.

As it has been said before this theorem gives a characterization of the renormalizable Lorenz maps in terms of the kneading pairs. Theorem $\mathrm{C}(\mathrm{c})$ is important because it gives a partial answer (at the symbolic level) to Conjecture 1.16(1-2) of $[15]$.

\section{Proof of Theorem B}

We start this section by proving some preliminary results and Proposition 2.10. Finally we will prove Theorem B.

The following lemma is due to Alsedà and Mañosas [4].

Lemma 3.1. The following statements hold:

(a) If $a=p / q$ with $(p, q)=1$ then $\widehat{\underline{I}}_{\varepsilon}(a)$ and $\widehat{\underline{I}}_{\delta}(a)$ are periodic with period $q$ (i.e. $S^{q}\left(\widehat{\underline{I}}_{\varepsilon}(a)\right)=\widehat{\underline{I}}_{\varepsilon}(a)$ and $\left.S^{q}\left(\underline{\widehat{I}}_{\delta}(a)\right)=\widehat{\underline{I}}_{\delta}(a)\right)$.

(b) Let $a, b \in \mathbb{R}$ with $a<b$. Then $\widehat{\widehat{I}}_{\varepsilon}(a)<\widehat{I}_{\varepsilon}(b), \widehat{I}_{\delta}(a)<\widehat{I}_{\delta}(b), \widehat{I}_{\varepsilon}^{*}(a)<\widehat{I}_{\varepsilon}^{*}(b)$ and $\widehat{\underline{I}}_{\delta}^{*}(a)<\widehat{I}_{\delta}^{*}(b)$.

From Theorem A and Proposition 2.3 we have the following. 
Lemma 3.2. Let $a \in \mathbb{R}$. Then $\widehat{\widehat{I}}_{\delta}^{*}(a), \underline{I}_{\varepsilon}(a) \in \mathcal{E}$ are minimal and $\widehat{\widehat{I}}_{\delta}(a), \widehat{I}_{\varepsilon}^{*}(a) \in \mathcal{E}_{\delta}$ are maximal.

Lemma 3.3. Let $a \in \mathbb{R}$. Then $\varepsilon_{1}(a) \leq \varepsilon_{i}(a) \leq \varepsilon_{1}(a)+1$ and $\delta_{1}(a)-1 \leq \delta_{i}(a) \leq$ $\delta_{1}(a)$ for all $i \geq 1$.

Proof. We recall that $\varepsilon_{i}(a)=E(i a)-E((i-1) a)=E(a+(i-1) a)-E((i-1) a)$. Then, from the fact that $E(x)+E(y) \leq E(x+y) \leq E(x)+E(y)+1$ for all $x, y \in \mathbb{R}$, we have that $\varepsilon_{1}(a) \leq \varepsilon_{i}(a) \leq \varepsilon_{1}(a)+1$ for all $i \geq 1$. In a similar way we can prove that $\delta_{1}(a)-1 \leq \delta_{i}(a) \leq \delta_{1}(a)$ for all $i \geq 1$.

Lemma 3.4. Let $a \in(0,1)$ Then $\widehat{\underline{I}}_{\varepsilon}(a), \widehat{\underline{I}}_{\delta}^{*}(a), \widehat{\underline{I}}_{\delta}(a), \widehat{\underline{I}}_{\varepsilon}^{*}(a) \in \Sigma_{2}$.

Proof. The statement follows from from Lemmas 3.1(a) and 2.8, the fact that $\varepsilon_{1}(a)=\delta_{1}(a)-1=E(a)=\widetilde{E}(a)$ for each $a \notin \mathbb{Z}$ and Lemma 3.3.

Now, from Lemmas 3.4 and 3.2 we obtain:

Corollary 3.5. Let $a \in(0,1)$. Then $\widehat{\widehat{I}}_{\varepsilon}(a), \widehat{\underline{I}}_{\delta}^{*}(a) \in \mathcal{E}_{\varepsilon}^{*}$ and $\widehat{\widehat{I}}_{\delta}(a), \underline{\underline{I}}_{\varepsilon}^{*}(a) \in \mathcal{E}_{\delta}^{*}$.

Lemma 3.6. Let $a \in \mathbb{Q}^{*}$ be with $(p, q)=1$. Then $\varepsilon_{q}(a)=\varepsilon_{1}(a)+1$.

Proof. If $\varepsilon_{q}(a) \neq \varepsilon_{1}(a)+1$ then, by Lemma 3.3, we can assume that $\varepsilon_{q}(a)=\varepsilon_{1}(a)$. By Lemma 3.1(a), $\widehat{\underline{I}}_{\varepsilon}(a)=\left(\varepsilon_{1}(a) \underline{r}(a) \varepsilon_{1}(a)\right)^{\infty}$ and, by Lemma 3.2,

$$
S^{q-1}\left(\widehat{\underline{I}}_{\varepsilon}(a)\right)=\left(\varepsilon_{1}(a) \varepsilon_{1}(a) \underline{r}(a)\right)^{\infty} \geq \widehat{\underline{I}}_{\varepsilon}(a) .
$$

Thus, by Lemma 3.3, $\varepsilon_{2}(a)=\varepsilon_{1}(a)$ and, proceeding inductively, we obtain that $\widehat{I}_{\varepsilon}(a)=\left(\varepsilon_{1}(a)\right)^{\infty} ;$ a contradiction by Lemma 3.1(a).

The following remark already allows us to prove Proposition 2.10 .

Remark 3.7. For each $a \in \mathbb{Q}^{*}$, in view of Lemmas 2.8 and 3.6, we can write

$$
\begin{aligned}
& \widehat{\underline{I}}_{\delta}^{*}(a)=\varepsilon_{1}(a) \underline{r}(a)\left(\varepsilon_{1}(a)\left(\varepsilon_{1}(a)+1\right) \underline{r}(a)\right)^{\infty}, \\
& \widehat{\underline{I}}_{\varepsilon}(a)=\left(\varepsilon_{1}(a) \underline{r}(a)\left(\varepsilon_{1}(a)+1\right)\right)^{\infty}, \\
& \widehat{\underline{I}}_{\delta}(a)=\left(\left(\varepsilon_{1}(a)+1\right) \underline{r}(a) \varepsilon_{1}(a)\right)^{\infty}, \text { and } \\
& \widehat{\underline{I}}_{\varepsilon}^{*}(a)=\left(\varepsilon_{1}(a)+1\right) \underline{r}(a)\left(\left(\varepsilon_{1}(a)+1\right) \varepsilon_{1}(a) \underline{r}(a)\right)^{\infty} .
\end{aligned}
$$

Proof of Proposition 2.10. It follows from Remark 3.7 and the fact that $\varepsilon_{1}(a)=$ 0.

Lemma 3.8. Let $a=p / q \in \mathbb{Q}^{*}$ be with $(p, q)=1$. Then the following statements hold

(a) $\varepsilon_{1}(a)\left(\varepsilon_{1}(a)+1\right) \underline{r}(a)>\varepsilon_{1}(a) \underline{r}(a) \varepsilon_{1}(a)$, and $\left(\varepsilon_{1}(a)+1\right) \varepsilon_{1}(a) \underline{r}(a)<\left(\varepsilon_{1}(a)+1\right) \underline{r}(a)\left(\varepsilon_{1}(a)+1\right)$.

(b) For $1<j \leq q-1$ we have $\varepsilon_{j}(a) \ldots \varepsilon_{q-1}(a) \varepsilon_{1}(a)\left(\varepsilon_{1}(a)+1\right) \varepsilon_{2}(a) \ldots \varepsilon_{j-1}(a)>\varepsilon_{1}(a) \underline{r}(a) \varepsilon_{1}(a)$, $\varepsilon_{j}(a) \ldots \varepsilon_{q-1}(a)\left(\varepsilon_{1}(a)+1\right) \varepsilon_{1}(a) \varepsilon_{2}(a) \ldots \varepsilon_{j-1}(a)>\varepsilon_{1}(a) \underline{r}(a)\left(\varepsilon_{1}(a)+1\right)$, $\varepsilon_{j}(a) \ldots \varepsilon_{q-1}(a)\left(\varepsilon_{1}(a)+1\right) \varepsilon_{1}(a) \varepsilon_{2}(a) \ldots \varepsilon_{j-1}(a)<\left(\varepsilon_{1}(a)+1\right) \underline{r}(a)\left(\varepsilon_{1}(a)+1\right)$, and $\varepsilon_{j}(a) \ldots \varepsilon_{q-1}(a) \varepsilon_{1}(a)\left(\varepsilon_{1}(a)+1\right) \varepsilon_{2}(a) \ldots \varepsilon_{j-1}(a)<\left(\varepsilon_{1}(a)+1\right) \underline{r}(a) \varepsilon_{1}(a)$ 
Proof. Since, by Remark 3.7 and Lemma 3.2, we have that

$$
\widehat{\underline{I}}_{\delta}^{*}(a)=\varepsilon_{1}(a) \underline{r}(a)\left(\varepsilon_{1}(a)\left(\varepsilon_{1}(a)+1\right) \underline{r}(a)\right)^{\infty}
$$

and it is minimal, then $\varepsilon_{1}(a)\left(\varepsilon_{1}(a)+1\right) \underline{r}(a) \geq \varepsilon_{1}(a) \underline{r}(a) \varepsilon_{1}(a)$. If $\varepsilon_{1}(a)\left(\varepsilon_{1}(a)+\right.$ 1) $\underline{r}(a)=\varepsilon_{1}(a) \underline{r}(a) \varepsilon_{1}(a)$, then

$\underline{\underline{I}}_{\delta}^{*}(a)=\varepsilon_{1}(a) \underline{r}(a) \varepsilon_{1}(a)\left(\varepsilon_{1}(a)+1\right) \cdots>\varepsilon_{1}(a)\left(\varepsilon_{1}(a)+1\right) \underline{r}(a) \varepsilon_{1}(a) \cdots=S^{q-1}\left(\widehat{I}_{\delta}^{*}(a)\right)$;

a contradiction with the minimality of $\widehat{\underline{I}}_{\delta}^{*}(a)$. This ends the proof of the first inequality of (a). Now we prove the first inequality of (b). Again by the minimality of $\widehat{\underline{I}}_{\delta}^{*}(a)$, for $1<j \leq q-1$, we have $\varepsilon_{j}(a) \ldots \varepsilon_{q-1}(a) \varepsilon_{1}(a)\left(\varepsilon_{1}(a)+1\right) \varepsilon_{2}(a) \ldots \varepsilon_{j-1}(a) \geq$ $\varepsilon_{1}(a) \underline{r}(a) \varepsilon_{1}(a)$. If the equality holds, then we have

$$
\begin{aligned}
S^{j-1}\left(\widehat{I}_{\delta}^{*}(a)\right) & =\varepsilon_{j}(a) \ldots \varepsilon_{q-1}(a) \varepsilon_{1}(a)\left(\varepsilon_{1}(a)+1\right) \varepsilon_{2}(a) \ldots \varepsilon_{j-1}(a) \varepsilon_{j}(a) \ldots \\
& =\varepsilon_{1}(a) \underline{r}(a) \varepsilon_{1}(a) \varepsilon_{1}(a) \cdots<\varepsilon_{1}(a) \underline{r}(a) \varepsilon_{1}(a)\left(\varepsilon_{1}(a)+1\right) \underline{r}(a) \ldots \\
& =\widehat{\underline{I}}_{\delta}^{*}(a)
\end{aligned}
$$

a contradiction. Hence,

$$
\varepsilon_{j}(a) \ldots \varepsilon_{q-1}(a) \varepsilon_{1}(a)\left(\varepsilon_{1}(a)+1\right) \varepsilon_{2}(a) \ldots \varepsilon_{j-1}(a)>\varepsilon_{1}(a) \underline{r}(a) \varepsilon_{1}(a) .
$$

To prove the second inequality of (b) note that, by Remark 3.7 and Lemma 3.2, we have that

$$
\widehat{\underline{I}}_{\varepsilon}(a)=\left(\varepsilon_{1}(a) \underline{r}(a)\left(\varepsilon_{1}(a)+1\right)\right)^{\infty}
$$

and it is periodic of period $q$ and minimal. Then, for $1<j \leq q-1$, we have $S^{j-1}\left(\widehat{I}_{\varepsilon}(a)\right)>\underline{I}_{\varepsilon}(a)$. Thus,

$$
\varepsilon_{j}(a) \ldots \varepsilon_{q-1}(a)\left(\varepsilon_{1}(a)+1\right) \varepsilon_{1}(a) \varepsilon_{2}(a) \ldots \varepsilon_{j-1}(a)>\varepsilon_{1}(a) \underline{r}(a)\left(\varepsilon_{1}(a)+1\right)
$$

since otherwise the equality holds and, consequently, $S^{j-1}\left(\widehat{\underline{I}}_{\varepsilon}(a)\right)=\widehat{\underline{I}}_{\varepsilon}(a)$ with $j<$ $q$; a contradiction. This concludes the proof of the second inequality of statement (b). The remaining inequalities of the lemma follow in a similar way by using the sequences $\widehat{\underline{I}}_{\varepsilon}^{*}(a)$ and $\widehat{\underline{I}}_{\delta}(a)$ instead of $\widehat{\underline{I}}_{\delta}^{*}(a)$ and $\widehat{\underline{I}}_{\varepsilon}(a)$.

Proof of Theorem B. We start proving (a). Set $\underline{\alpha}=\alpha_{1} \alpha_{2} \ldots$ and $\underline{\beta}=\beta_{1} \beta_{2} \ldots$ Since $\underline{\alpha}<\underline{\beta}$, there exists $k \geq 1$ such that $\alpha_{1} \alpha_{2} \ldots \alpha_{k-1}=\beta_{1} \beta_{2} \ldots \beta_{k-1}$ and $\alpha_{k}<\beta_{k}$. Then, from the definition of the $\odot-$ product it follows that $a \odot \underline{\alpha}<a \odot \underline{\beta}$. We note that in particular, from Proposition 2.10, we have proved that

$$
\widehat{\underline{I}}_{\delta}^{*}(a)=a \odot 0^{\infty} \leq a \odot \underline{\alpha} \leq \overline{a \odot 1^{\infty}}=\widehat{\underline{I}}_{\varepsilon}(a)
$$

whenever $\alpha_{1}=0$ and

$$
\widehat{\underline{I}}_{\delta}(a)=\overline{a \odot 0^{\infty}} \leq a \odot \underline{\alpha} \leq a \odot 1^{\infty}=\widehat{\underline{I}}_{\varepsilon}^{*}(a)
$$

whenever $\alpha_{1}=1$. This ends the proof of (a).

Now we prove (b). We only will prove the first statement of (b). The second one follows similarly. From Theorem 2.7 and Lemma 3.2 we have $a \odot 0^{\infty} \in \mathcal{E}_{\varepsilon}^{*} \subset \mathcal{E}_{\varepsilon}$. Therefore, we may assume that $\underline{\alpha}=\alpha_{1} \alpha_{2} \ldots \neq 0^{\infty}$. By Remark 2.4 we have $\alpha_{1}=0$. Consequently, $a \odot \underline{\alpha}=0 \underline{r}(a) 01 \underline{r}(a) \alpha_{2} \widehat{\alpha}_{2} \underline{r}(a) \ldots$. To end the proof statement (b) we have to prove that $S^{j}(a \odot \underline{\alpha}) \geq a \odot \underline{\alpha}$ for each $j \geq 1$. Let $a=p / q$ with $(p, q)=1$ and $m \geq 1$. Then

$$
S^{q m}(a \odot \underline{\alpha})=\widehat{\alpha}_{m} \underline{r}(a) \alpha_{m+1} \widehat{\alpha}_{m+1} \ldots .
$$


If $\alpha_{m}=1$, then $\widehat{\alpha}_{m}=0$ and, since $\underline{\alpha}$ is minimal, we have $S^{q m}(a \odot \underline{\alpha}) \geq a \odot \underline{\alpha}$. If $\alpha_{m}=0$ and $\widehat{\alpha}_{m}=1$ then clearly, we are done. Now we look at

$$
S^{m q-1}(a \odot \underline{\alpha})=\alpha_{m} \widehat{\alpha}_{m} \underline{r}(a) \alpha_{m+1} \widehat{\alpha}_{m+1} \ldots .
$$

If $\alpha_{m}=1$, obviously $S^{m q-1}(a \odot \underline{\alpha}) \geq a \odot \underline{\alpha}$. Assume that $\alpha_{m}=0$. Then $\alpha_{m} \widehat{\alpha}_{m}=01$ and the desired inequality follows from Lemma 3.8(a) (recall that $a \in \mathbb{Q}^{*}$; that is $\left.\varepsilon_{1}(a)=0\right)$. Now, assume that $1<j \leq q-1$. Then

$$
S^{(m-1) q+j-1}(a \odot \underline{\alpha})=\varepsilon_{j}(a) \ldots \varepsilon_{q-1}(a) \alpha_{m} \widehat{\alpha}_{m} \ldots
$$

and, from Lemma 3.8(b), we get $S^{(m-1) q+j-1}(a \odot \underline{\alpha}) \geq a \odot \underline{\alpha}$. This ends the proof of statement (b).

Finally we prove (c). Assume that $a=p / q$ with $(p, q)=1$ and set $\underline{\alpha}=\alpha_{1} \alpha_{2} \ldots$, $\underline{\beta}=\beta_{1} \beta_{2} \ldots$ and $\underline{\kappa}=\kappa_{1} \kappa_{2} \ldots$ Since $S^{n}(\underline{\alpha}) \leq \underline{\kappa}$, we obtain $S^{n}(a \odot \underline{\alpha}) \leq a \odot \underline{\kappa}$ in a similar way as above by using Lemma 3.8(c) instead of Lemma 3.8(a) and Lemma 3.8(d) instead of Lemma 3.8(b). On the other hand, from $S^{n}(\beta) \geq \underline{\kappa}$ and Lemma 3.8(a)-(b) we obtain $S^{n}(a \odot \underline{\beta}) \geq a \odot \underline{\kappa}$. This ends the proof of the theorem.

\section{Proof of Theorem C}

To prove Theorem $\mathrm{C}$ we need some technical results.

Lemma 4.1. The sets $\mathcal{E}^{*}, \mathcal{E}_{\varepsilon}$ and $\mathcal{E}_{\delta}$ are closed. Moreover, the set $\mathcal{E}_{0,1} \cap \mathcal{E}^{*}$ is compact in $\Sigma_{2} \times \Sigma_{2}$.

Proof. Take $\left\{\left(\underline{\nu}_{n}^{1}, \underline{\nu}_{n}^{2}\right)\right\}_{n \in \mathbb{N}}$, a sequence in $\mathcal{E}^{*}$ which converges to $\left(\underline{\nu}^{1}, \underline{\nu}^{2}\right)$. Clearly $\left(\underline{\nu}^{1}, \underline{\nu}^{2}\right) \in \mathcal{A D} \times \mathcal{A D}, \underline{\nu}_{1}$ and $\underline{\nu}_{2}$ are infinite and $\left(\underline{\nu}^{1}, \underline{\nu}^{2}\right)$ verifies property (KP1). Since each $\left(\underline{\nu}_{n}^{1}, \underline{\nu}_{n}^{2}\right)$ verifies property (KP2) we have that $\underline{\nu}_{n}^{1} \leq S^{j}\left(\underline{\nu}_{n}^{i}\right) \leq \underline{\nu}_{n}^{2}$ for all $j \geq 0, n \in \mathbb{N}$ and $i \in\{1,2\}$. In view of the continuity of $S$, by taking limits, we have $\underline{\nu}^{1} \leq S^{j}\left(\underline{\nu}^{i}\right) \leq \underline{\nu}^{2}$ for all $j \geq 0$ and $i \in\{1,2\}$ (recall that the order topology is compatible with the metric topology). Thus, $\left(\underline{\nu}^{1}, \underline{\nu}^{2}\right) \in \mathcal{E}^{*}$. The facts that $\mathcal{E}_{\varepsilon}$ and $\mathcal{E}_{\delta}$ are closed follow in a similar way by using Proposition 2.3. Finally, in view of Remark 2.4 it follows that $1^{\infty}$ is an isolated point of $\mathcal{E}_{\varepsilon}$ and $0^{\infty}$ is isolated in $\mathcal{E}_{\delta}$. Therefore,

$$
\mathcal{E}_{0,1} \cap \mathcal{E}^{*}=\left(\mathcal{E}_{\varepsilon}^{*} \times \mathcal{E}_{\delta}^{*}\right) \cap \mathcal{E}^{*}=\left(\Sigma_{2} \times \Sigma_{2}\right) \cap\left(\left(\left(\mathcal{E}_{\varepsilon} \backslash\left\{1^{\infty}\right\}\right) \times\left(\mathcal{E}_{\delta} \backslash\left\{0^{\infty}\right\}\right)\right) \cap \mathcal{E}^{*}\right)
$$

is compact.

In the next lemma and in the proof of Theorem $\mathrm{C}$ we will use the following notation. We will use the symbol $s$ to denote,+- or $\emptyset$. So, for $x \in \mathbb{R}, x^{s}$ will stand for $x^{+}, x^{-}$or $x$, respectively. Also, if $J$ is a closed interval from $\mathbb{R}$ such that $0 \in \operatorname{Int}(J)$, when writing $x^{s} \in J$ we will mean that $x \in J$ but $x^{s} \notin$ $\left\{(\min J)^{-},(\max J)^{+}, 0\right\}$. Finally, for $x^{s} \in J$ we set

$$
\chi\left(x^{s}\right)= \begin{cases}0 & \text { if } x>0 \text { or } x^{s}=0^{+} \\ 1 & \text { if } x<0 \text { or } x^{s}=0^{-}\end{cases}
$$

and $\widehat{\chi}\left(x^{s}\right)=\widehat{\chi\left(x^{s}\right)}=1-\chi\left(x^{s}\right)$.

Lemma 4.2. Let $F \in \mathcal{L} m$ be such that $R_{F}=\{p / q\}$ with $p \in \mathbb{Z}, q \in \mathbb{N}$ and $(p, q)=1$, and let $J$ be a closed interval of $\mathbb{R}$ obtained from $F$ as in the definition of a renormalizable map. Assume that $0 \in \operatorname{Int}(J)$ and set $G=F^{q}-p$. Then the following statements hold 
(a) $\widehat{\underline{I}}_{F}(\max J)=\underline{\underline{I}}_{\varepsilon}(a)=0 \underline{r}(a)(10 \underline{r}(a))^{\infty}$, and $\underline{\widehat{I}}_{F}(\min J)=\underline{\widehat{I}}_{\delta}(a)=1 \underline{r}(a)(01 \underline{r}(a))^{\infty}$.

(b) For each $z \in J$ and $s \in\{+,-, \emptyset\}$ such that $z^{s} \in J$ we have

$$
\widehat{\underline{I}}_{F}\left(z^{s}\right)=\chi\left(z^{s}\right) \underline{r}(a) d\left(z^{s}\right) \widehat{\underline{I}}_{F}\left(\left(G\left(z^{s}\right)\right)^{s}\right)
$$

with $d\left(z^{s}\right) \in\{0,1\}$. In particular, $\widehat{\underline{I}}_{F}\left(0^{-}\right)=1 \underline{r}(a) d\left(0^{-}\right) \widehat{\underline{I}}_{F}\left(\left(G\left(0^{-}\right)\right)^{-}\right)$and $\widehat{\underline{I}}_{F}\left(0^{+}\right)=0 \underline{r}(a) d\left(0^{+}\right) \widehat{\underline{I}}_{F}\left(\left(G\left(0^{+}\right)\right)^{+}\right)$.

(c) Assume that $\min J \leq G\left(0^{+}\right)<0<G\left(0^{-}\right) \leq \max J$. Then, for each $z \in J$ and $s \in\{+,-, \emptyset\}$ such that $z^{s},\left(G\left(z^{s}\right)\right)^{s} \in \bar{J}$ we have

$$
\widehat{\underline{I}}_{F}\left(z^{s}\right)=\chi\left(z^{s}\right) \underline{r}(a) \widehat{\chi}\left(\left(G\left(z^{s}\right)\right)^{s}\right) \widehat{\underline{I}}_{F}\left(\left(G\left(z^{s}\right)\right)^{s}\right) .
$$

In particular, $\widehat{\underline{I}}_{F}\left(0^{-}\right)=1 \underline{r}(a) 10 \underline{r}(a) \cdots$ and $\widehat{\widehat{I}}_{F}\left(0^{+}\right)=0 \underline{r}(a) 01 \underline{r}(a) \cdots$.

Proof. Statement (a) follows from the definition of $J$, from the fact that $0 \in \operatorname{Int}(J)$, [4, Lemma 4.4] and Proposition 2.10. Now we prove (b). From Theorem 2.7 and Proposition 2.10 we have

$$
\begin{aligned}
& \widehat{I}_{\delta}(a)=1 \underline{r}(a) 01 \underline{r}(a) 01 \underline{r}(a) \cdots \leq \widehat{I}_{F}\left(0^{-}\right) \leq \widehat{I}_{\varepsilon}^{*}(a)=1 \underline{r}(a) 10 \underline{r}(a) 10 \underline{r}(a) \cdots \\
& \widehat{\underline{I}}_{\delta}^{*}(a)=0 \underline{r}(a) 01 \underline{r}(a) 01 \underline{r}(a) \cdots \leq \widehat{I}_{F}\left(0^{+}\right) \leq \widehat{\widehat{I}}_{\varepsilon}(a)=0 \underline{r}(a) 10 \underline{r}(a) 10 \underline{r}(a) \cdots .
\end{aligned}
$$

Thus

$$
\widehat{\underline{I}}_{F}\left(0^{+}\right)=0 \underline{r}(a) \cdots \quad \text { and } \quad \widehat{\widehat{I}}_{F}\left(0^{-}\right)=1 \underline{r}(a) \cdots .
$$

Let $x \in(\min J, \max J) \backslash\{0\}$. From [5, Lemma 1] it follows easily that

$$
\begin{array}{ll}
\widehat{I}_{F}\left(0^{+}\right) \leq \widehat{I}_{F}(x) \leq \widehat{I}_{F}(\max J) & \text { if } x>0, \text { and } \\
\widehat{I}_{F}(\min J) \leq \widehat{I}_{F}(x) \leq \widehat{I}_{F}\left(0^{-}\right) & \text {if } x<0 .
\end{array}
$$

Therefore, from (4.1) and (a) we have

$$
\widehat{\underline{I}}_{F}(x)=0 \underline{r}(a) \cdots \text { if } x>0 \quad \text { and } \quad \widehat{\underline{I}}_{F}(x)=1 \underline{r}(a) \cdots \text { if } x<0 .
$$

This proves (b).

Now we prove (c). We will start by showing that $\widehat{\widehat{I}}_{F}\left(0^{-}\right)=1 \underline{r}(a) 10 \underline{r}(a) \cdots$ and $\widehat{\underline{I}}_{F}\left(0^{+}\right)=0 \underline{r}(a) 01 \underline{r}(a) \cdots$. Since $\min J \leq G\left(0^{+}\right)<0<G\left(0^{-}\right) \leq \max J$, from (b) it follows that $\widehat{\underline{I}}_{F}\left(\left(G\left(0^{-}\right)\right)^{-}\right)=0 \underline{r}(a) \cdots$ and $\widehat{\underline{I}}_{F}\left(\left(G\left(0^{+}\right)\right)^{+}\right)=1 \underline{r}(a) \cdots$. Hence,

$$
\widehat{\underline{I}}_{F}\left(0^{-}\right)=1 \underline{r}(a) d\left(0^{-}\right) 0 \underline{r}(a) \cdots \quad \text { and } \quad \widehat{\underline{I}}_{F}\left(0^{+}\right)=0 \underline{r}(a) d\left(0^{+}\right) \underline{1} \underline{r}(a) \cdots .
$$

Note that $\widehat{\underline{I}}_{\delta}(a) \leq \widehat{\underline{I}}_{F}\left(0^{-}\right)$and $\widehat{\underline{I}}_{F}\left(0^{+}\right) \leq \widehat{\widehat{I}}_{\varepsilon}(a)$ and, consequently, $d\left(0^{-}\right)=1$ and $d\left(0^{+}\right)=0$.

Now we prove the first statement of (c). Since $\left(G\left(z^{s}\right)\right)^{s} \in J$, from (b) we have

$$
\widehat{\underline{I}}_{F}\left(z^{s}\right)=\chi\left(z^{s}\right) \underline{r}(a) d\left(z^{s}\right) \chi\left(\left(G\left(z^{s}\right)\right)^{s}\right) \underline{r}(a) \cdots .
$$

Therefore, we have to see that $d\left(z^{s}\right) \chi\left(\left(G\left(z^{s}\right)\right)^{s}\right) \notin\{00,11\}$. Assume that $\chi\left(z^{s}\right)=1$. From the definition of $\chi$ and (4.2) it follows that $\widehat{I}_{F}(\min J) \leq \widehat{I}_{F}\left(z^{s}\right) \leq \widehat{I}_{F}\left(0^{-}\right)$. Therefore, from (a) and the part of (c) already proved, we get

$$
1 \underline{r}(a) 01 \underline{r}(a) \cdots \leq 1 \underline{r}(a) d\left(z^{s}\right) \chi\left(\left(G\left(z^{s}\right)\right)^{s}\right) \underline{r}(a) \cdots \leq 1 \underline{r}(a) 10 \underline{r}(a) \cdots,
$$


which shows that $d\left(z^{s}\right) \chi\left(\left(G\left(z^{s}\right)\right)^{s}\right) \notin\{00,11\}$ when $\chi\left(z^{s}\right)=1$. If $\chi\left(z^{s}\right)=0$ then we obtain $d\left(z^{s}\right) \chi\left(\left(G\left(z^{s}\right)\right)^{s}\right) \notin\{00,11\}$ in a similar way. This ends the proof of the lemma.

Proof of Theorem $C$. We start by proving (a). We have to show that $\widehat{I}_{F}\left(0^{+}\right)=$ $\left.a \odot \widehat{\underline{I}}_{\mathcal{R}_{a}(F)}\left(0^{+}\right)\right)$and $\left.\widehat{\underline{I}}_{F}\left(0^{-}\right)=a \odot \widehat{\underline{I}}_{\mathcal{R}_{a}(F)}\left(0^{-}\right)\right)$. Since $F$ is renormalizable, we have $\min J \leq G\left(0^{+}\right)<0<G\left(0^{-}\right) \leq \max J$ and $G^{i}\left(0^{-}\right), G^{i}\left(0^{+}\right) \in\left[G\left(0^{+}\right), G\left(0^{-}\right)\right] \subset$ $[\min J, \max J]$ for each $i \in \mathbb{N}$. Therefore, from the iterative use of Lemma 4.2(c) we obtain

$$
\begin{aligned}
& \widehat{I}_{F}\left(0^{-}\right)=1 \underline{r}(a) 10 \underline{r}(a) d_{2}^{-} \widehat{d_{2}^{-}} \underline{r}(a) d_{3}^{-} \widehat{d_{3}^{-}} \ldots \\
& \widehat{I}_{F}\left(0^{+}\right)=0 \underline{r}(a) 01 \underline{r}(a) d_{2}^{+} \widehat{d_{2}^{+}} \underline{r}(a) d_{3}^{+} \widehat{d_{3}^{+}} \ldots
\end{aligned}
$$

where, for $i=2,3, \ldots$ we have that $d_{i}^{-}$(respectively $d_{i}^{+}$) is 1 if $G^{i}\left(0^{-}\right)>0$ (respectively $G^{i}\left(0^{+}\right) \geq 0$ ) and 0 if $G^{i}\left(0^{-}\right) \leq 0$ (respectively $\left.G^{i}\left(0^{+}\right)<0\right)$. So,

$$
\widehat{\underline{I}}_{F}\left(0^{-}\right)=a \odot\left(1 d_{2}^{-} d_{3}^{-} \cdots\right) \quad \text { and } \quad \widehat{\underline{I}}_{F}\left(0^{+}\right)=a \odot\left(0 d_{2}^{+} d_{3}^{+} \cdots\right) .
$$

On the other hand,

$$
\widehat{\underline{I}}_{\mathcal{R}_{a}(F)}\left(0^{-}\right)=1 d_{2}^{-} d_{3}^{-} \ldots \quad \text { and } \quad \widehat{\underline{I}}_{\mathcal{R}_{a(F)}}\left(0^{+}\right)=0 d_{2}^{+} d_{3}^{+} \ldots
$$

(observe that $G\left(0^{-}\right)$and $G\left(0^{+}\right)$play the role of $0^{-}$and $0^{+}$for $\mathcal{R}_{a}(F)$, respectively). This ends the proof of (a).

To prove (b) we start by taking $J$ as in the definition of a renormalizable map. Since $\mathcal{K}(F)=a \odot(\underline{\alpha}, \underline{\beta}), \widehat{\underline{I}}_{F}\left(0^{+}\right)=a \odot \underline{\alpha}$ and $\widehat{\underline{I}}_{F}\left(0^{-}\right)=a \odot \underline{\beta}$. Thus, since $(\underline{\alpha}, \underline{\beta}) \in$ $\mathcal{E}_{0,1}$, by Proposition 2.10 and Remark 2.4 we have

$$
\widehat{\underline{I}}_{F}\left(0^{+}\right)=a \odot \underline{\alpha}=0 \underline{r}(a) 01 \underline{r}(a) \cdots<0 \underline{r}(a)(10 \underline{r}(a))^{\infty}=\overline{a \odot 1^{\infty}}=\widehat{\underline{I}}_{\varepsilon}(a)
$$

and, similarly, $\widehat{I}_{F}\left(0^{-}\right)>\underline{I}_{\delta}(a)$. On the other hand, from [4, Lemma 4.4], it follows that if $\min J=0$ (respectively, $\max J=0$ ) then $\widehat{\underline{I}}_{F}\left(0^{-}\right)=\widehat{I}_{\delta}(a)$ (respectively, $\left.\widehat{I}_{F}\left(0^{+}\right)=\widehat{I}_{\varepsilon}(a)\right)$. In consequence, $\min J<0<\max J$.

Now, to prove (b) it suffices to show that $\min J \leq G\left(0^{+}\right)<0<G\left(0^{-}\right) \leq \max J$ and $G\left(\left[G\left(0^{+}\right), G\left(0^{-}\right)\right]\right) \subset\left[G\left(0^{+}\right), G\left(0^{-}\right)\right]$. Note that, since $G$ is increasing and continuous on $[\min J, 0)$ and $(0, \max J]$ it is enough to show that min $J \leq G\left(0^{+}\right)<$ $0<G\left(0^{-}\right) \leq \max J, G\left(0^{+}\right) \leq G\left(G\left(0^{+}\right)\right)$and $G\left(G\left(0^{-}\right)\right) \leq G\left(0^{-}\right)$. We only will prove the statements about $G\left(0^{-}\right)$. The other ones follow similarly.

First we will prove that $0<G\left(0^{-}\right) \leq \max J$. Observe that if $\underline{\beta}=\beta_{1} \beta_{2} \ldots$ then $\widehat{\underline{I}}_{F}\left(0^{-}\right)=\beta_{1} \underline{r}(a) \beta_{1} \widehat{\beta}_{1} \underline{r}(a) \cdots$. Consequently, by Lemma $4.2(\mathrm{~b})$,

$$
\widehat{\underline{I}}_{F}\left(0^{-}\right)=1 \underline{r}(a) 1 \underline{\underline{I}}_{F}\left(\left(G\left(0^{-}\right)\right)^{-}\right) \quad \text { with } \quad \widehat{\underline{I}}_{F}\left(\left(G\left(0^{-}\right)\right)^{-}\right)=0 \underline{r}(a) \beta_{2} \widehat{\beta}_{2} \underline{r}(a) \cdots .
$$

Note that $G\left(0^{-}\right) \neq 0$. Otherwise, by Theorem 2.7, $\widehat{I}_{F}\left(\left(G\left(0^{-}\right)\right)^{-}\right)=\widehat{I}_{F}\left(0^{-}\right) \geq$ $\widehat{I}_{\delta}(a)=1 \cdots ;$ a contradiction.

Assume that $G\left(0^{-}\right)<0$. Since $G(\min J)=\min J$ and $G$ is increasing in [ $\left.\min J, 0\right)$ we have $G\left(0^{-}\right) \geq \min J$. Then, by Lemma $4.2(\mathrm{~b}), \widehat{\underline{I}}_{F}\left(\left(G\left(0^{-}\right)\right)^{-}\right)$starts with 1 ; a contradiction. Thus, $G\left(0^{-}\right)>0$. Assume now that $G\left(0^{-}\right)>\max J$. By Lemma $4.2(\mathrm{a})$ we know that $0 \underline{r}(a) 10 \underline{r}(a) 10 \underline{r}(a) \cdots=\widehat{\underline{I}}_{\varepsilon}(a)=\widehat{\underline{I}}_{F}(\max J)$. Moreover, from [5, Lemma 1] (since $F$ is symbolically expansive and $G\left(0^{-}\right)>\max J$ ) it 
follows that $\widehat{\underline{I}}_{F}(\max J)<\widehat{I}_{F}\left(G\left(0^{-}\right)\right)$. Thus, since $\widehat{\underline{I}}_{F}(\max J)$ is infinite, it follows that $\widehat{\underline{I}}_{F}(\max J)<\widehat{\underline{I}}_{F}\left(\left(G\left(0^{-}\right)\right)^{-}\right)$and, consequently,

$$
0 \underline{r}(a) 10 \underline{r}(a) 10 \underline{r}(a) \cdots<0 \underline{r}(a) \beta_{2} \widehat{\beta}_{2} \underline{r}(a) \cdots .
$$

So, there exists $i \geq 2$ such that $\beta_{i} \widehat{\beta}_{i}=11$; a contradiction. Hence, we have proved that $0<G\left(0^{-}\right) \leq \max J$.

Now we will prove that $G\left(G\left(0^{-}\right)\right) \leq G\left(0^{-}\right)$. To this end we assume that $G\left(G\left(0^{-}\right)\right)>G\left(0^{-}\right)$. Then, there exists $0<z<G\left(0^{-}\right)$such that $G(z)=z$. Thus, $G([z, \max J])=[z, \max J]$ and, consequently, $G^{i}(x) \in[z, \max J]$ for each $i \geq 0$ and $x \in[z, \max J]$. Therefore, by Lemma $4.2(\mathrm{a}, \mathrm{c})$ we see that

$$
\widehat{\underline{I}}_{F}(x)=0 \underline{r}(a) 10 \underline{r}(a) 10 \cdots=\widehat{\underline{I}}_{\varepsilon}(a)=\widehat{\underline{I}}_{F}(\max J)
$$

for each $x \in[z, \max J]$. This contradicts the fact that $F$ is symbolically expansive and, hence, $G\left(G\left(0^{-}\right)\right) \leq G\left(0^{-}\right)$. This ends the proof of (b).

Now we prove (c). Let $a \in \mathbb{Q}^{*}$ and $\underline{\alpha}, \beta \in \mathcal{A D}_{0,1}$. Set $\underline{\alpha}=\alpha_{1} \alpha_{2} \ldots$ and $\underline{\beta}=\beta_{1} \beta_{2} \ldots$ and suppose that $\alpha_{1}=\beta_{1}$. Then

$$
\begin{aligned}
d(a \odot \underline{\alpha}, a \odot \underline{\beta}) & =\sum_{i=2}^{\infty}\left(\frac{1}{2^{i q}}\left|\alpha_{i}-\beta_{i}\right|+\frac{1}{2^{i q+1}}\left|\widehat{\alpha}_{i}-\widehat{\beta}_{i}\right|\right) \\
& \leq \frac{1}{2^{q-1}} \sum_{i=2}^{\infty} \frac{1}{2^{i}}\left|\alpha_{i}-\beta_{i}\right|=\frac{1}{2^{q-1}} d(\underline{\alpha}, \underline{\beta}) \leq \frac{1}{2} d(\underline{\alpha}, \underline{\beta}) .
\end{aligned}
$$

Thus, $d(\underline{\mathbf{a}} \odot \underline{\alpha}, \underline{\mathbf{a}} \odot \underline{\beta}) \leq \frac{1}{2|\underline{\mathbf{a}}|} d(\underline{\alpha}, \underline{\beta})$ for each $\underline{\mathbf{a}} \in\left(\mathbb{Q}^{*}\right)^{n}$. Observe that by Lemma 4.1 and Corollary 2.11 we have that $\mathcal{E}_{0,1} \cap \mathcal{E}^{*}$ is compact and $\underline{\mathbf{a}} \odot\left(\mathcal{E}_{0,1} \cap \mathcal{E}^{*}\right) \subset \mathcal{E}_{0,1} \cap \mathcal{E}^{*}$. Moreover, if $\left(\underline{\alpha}^{\prime}, \underline{\beta}^{\prime}\right),\left(\underline{\alpha}^{\prime \prime}, \underline{\beta}^{\prime \prime}\right) \in \mathcal{E}_{0,1} \cap \mathcal{E}^{*}$ then, by Remark 2.4 ,

$$
d^{*}\left(\underline{\mathbf{a}} \odot\left(\underline{\alpha}^{\prime}, \underline{\beta}^{\prime}\right), \underline{\mathbf{a}} \odot\left(\underline{\alpha}^{\prime \prime}, \underline{\beta}^{\prime \prime}\right)\right) \leq \frac{1}{2^{|\underline{a}|}} d^{*}\left(\left(\underline{\alpha}^{\prime}, \underline{\beta}^{\prime}\right),\left(\underline{\alpha}^{\prime \prime}, \underline{\beta}^{\prime \prime}\right)\right) .
$$

This ends the proof of the theorem.

\section{REFERENCES}

[1] Alsedà Ll., Falcó A., A characterization of the kneading pair for bimodal degree one circle maps, Ann. Inst. Fourier, Grenoble, 47 (1997), 273-304.

[2] Alsedà Ll., Llibre J. and Misiurewicz M., Combinatorial dynamics and entropy in dimension one, Advanced Series in Nonlinear Dynamics 5, World Scientific, Singapore, 1993.

[3] Alsedà Ll., Llibre J., Misiurewicz M., Tresser Ch., Periods and entropy for Lorentz-like maps, Ann. Inst. Fourier, Grenoble, 39 (1989), 929-952.

[4] Alsedà Ll., Mañosas F., Kneading theory and rotation interval for a class of circle maps of degree one, Nonlinearity 3 (1990), 413-452.

[5] Alsedà Ll., Mañosas F., Kneading theory for a family of circle maps with one discontonuity, Acta Math. Univ. Comeniae Vol LXV, 1 (1996) 11-22.

[6] Collet P. Eckmann J. P., Iterated maps on the interval as dynamical systems, Progress in Physics, Birkhäuser, 1980.

[7] Falcó A., Bifurcations and symbolic dynamics for bimodal degree one circle maps: The Arnol'd tongues and the Devil's staircase, Ph. D. Thesis, Universitat Autònoma de Barcelona, 1995.

[8] Glendinning P., Topological conjugation of Lorenz maps by $\beta$-transformations, Math. Proc. Camb. Phil. Soc. 107 (1990), 401-413.

[9] Glendinning P., Sparrow C., Prime and renormalisable kneading invariants and the dynamics of expanding Lorenz maps, Physica D 62 (1993), 22-50.

[10] Gukenheimer J., A strange, strange attractor, The Hopf bifurcations and its applications (Eds. J. Marsden and M. McCracken), Appl. Math. Sci. 19, Springer-Verlag, 1976. 
[11] Gukenheimer J., Williams R. F., Structural stability of Lorenz attractors, Inst. Hautes Études Sci. Publ. Math. 50 (1979), 307-320.

[12] Hubbard J. H., Sparrow C., The Classification of Topologically Expansive Lorenz Maps, Comm. on Pure and Appl. Math. Vol XLIII (1990), 431-443.

[13] Lorenz E. N., Deterministic non-periodic flow, J. Atmost. Sci. 20 (1963), 130-141.

[14] Martens M., The periodic points of renormalization, Ann. of Math. 147 (1998), 543-584.

[15] Martens M., de Melo W., Universal models for Lorenz maps, Preprint, IMPA 1997.

[16] Milnor J. Thurston P., On iterated maps on the interval I, II "Dynamical Systems", Lecture Notes in Math 1342, Springer, 1988.

[17] Misiurewicz M., Rotation intervals for a class of maps of the real line into itself, Ergod. Th. \& Dynam. Sys. 6 (1986), 117-132.

[18] Morales C. A., Pujals E. R., Singular strange attractors on the boundary of Morse-Smale systems, Ann. scient. Éc. Norm. Sup. 30 (1997), 693-717.

[19] Poincaré H., Sur les curves définies par les équations differentielles, Oeuvres completes, vol 1, 137-158, Gauthiers-Villars, Paris, 1952.

[20] Rhodes F., Thompson C. L., Rotation numbers for monotone fuctions on the circle, J. London Math. Soc. 34 (1986), 360-368.

[21] Sparrow C. T., The Lorenz equations: Bifurcations, chaos and strange attrators, Appl. Math. Sci. 41, Springer-Verlag, 1982.

[22] Williams R. F., The structure of Lorenz attractors, Inst. Hautes Études Sci. Publ. Math. 50 (1979), 321-347.

Departament de Matemàtiques, Universitat Autònoma de Barcelona, 08193 CerdanyOLA DEL VALLÈs, SPAiN

Current address: Departament de m Matmàtica Aplicada I, Universitat Politècnica de Catalunya, Diagonal 647, 08028 Barcelona, Spain

E-mail address: alseda@mat.uab.es

Universidad Cardenal Herrera-CeU, Facultad de Ciencias Sociales Juridicas y de la Comunicación, Campus de Elche, Comissari 1, 03203 Elche-Elx, Spain

E-mail address: falco.el@ceu.es 